\title{
A Review of Polarization Dependence Applications for Asymmetric Waveguides Vertical Couplers in Compound Semiconductor Indium Phosphide
}

\author{
Chee-Wei Lee \\ Cavendish Laboratory, University of Cambridge, J.J. Thomson Avenue, Cambridge CB3 OHE, UK \\ Correspondence should be addressed to Chee-Wei Lee, cwl30@cam.ac.uk
}

Received 5 May 2011; Accepted 22 June 2011

Academic Editor: Vittorio M. N. Passaro

Copyright () 2011 Chee-Wei Lee. This is an open access article distributed under the Creative Commons Attribution License, which permits unrestricted use, distribution, and reproduction in any medium, provided the original work is properly cited.

\begin{abstract}
This paper reviews and presents a coherent approach to the design of compact vertical coupler (VC) in InP-based compound semiconductor with variable polarization dependence. As a polarization-independent (PI) coupler, the VC is shown to transfer light with more than $90 \%$ efficiency for both transverse-electric (TE) and transverse-magnetic (TM) polarizations. As a polarization-mode splitter (PMS), the VC is shown to preferentially couple TE or TM modes with a contrast ratio of up to $20 \mathrm{~dB}$. We further demonstrate the single-mesa VC, which simplifies the fabrication process and potentially could improve the process yield, and its integration with a multimode interferometer (MMI). The versatility makes the VC a compact and useful input-stage device that not only maximizes input/output coupling efficiency to small active devices but also provides a degree of polarization control before the actual device.
\end{abstract}

\section{Introduction}

The concept of photonic integrated circuit (PIC), a photonic chip with many devices and multifunctional capability, actually emerged in the 1960s, about the same period where integrated electronics started moving to the forefront of technology. However, advances in PIC are few and limited compared to its electronic counterpart. This is because a functional PIC usually requires many different devices, both active and passive, each of which may have a distinct functionality and requirement. In contrast, complicated electronic IC can be formed just by transistors and a few passive components. Furthermore, feedback circuitry in photonics is still in its infancy, whereas in electronics, it is widely implemented. Thus, this has inhibited stable and consistent performance over some large scale integration.

The rapid development in optical communication systems has accelerated the drive for more integrated photonic devices because of the advantages it can provide. PIC is able to reduce the system size and cost and improve the reliability as the number of discrete devices is reduced. Besides, it minimizes the number of fiber interconnections between the discrete devices, which is usually the main source of optical loss, especially if the photonic devices are small compared to the fiber. Also, since the devices are now very near to each other on the same substrate, the optical propagation loss could be reduced as well.

Despite the advantages that PIC can provide, the search for a universal platform is still an elusive goal. Monolithic integration is the preferred architecture of integration. Since multiple optical devices are to be integrated into a chip with minimum size and maximum functionality, the devices must be compatible with each other, and the integration platform must be able to support the different devices. Generally, the material used for monolithic integration must possess the following characteristics:

(i) it must support all passive (light guiding) and active optical functions (modulation, amplification, switching, and etc.) required for a particular circuit,

(ii) it must be able to provide material bandgap in telecommunication wavelengths (the $1.30-1.60 \mu \mathrm{m}$ wavelength window), 
(iii) it must demonstrate good electrical functionality for optoelectronic applications, and

(iv) it must be suited for miniaturization.

One of the most commonly used materials that fulfill all the above requirements is the compound semiconductor indium phosphide (InP). Therefore, it will be used throughout our context.

Various photonic integrated devices have been demonstrated with InP. The simplest PIC demonstrated is the spot-size-converter integrated laser [1-5]. Lasers integrated with electroabsorption modulators (EAM) to provide higher bandwidth in excess of $1 \mathrm{~Gb} / \mathrm{s}$ have also been demonstrated since more than a decade ago [6-9]. They exceed the bandwidth achievable by the commercially available direct modulated laser for telecommunication. Following this, laser integrated with semiconductor optical amplifier (SOA) has been developed in 2002 [10]. PICs with higher integration level, with some having over 50 functions on a single chip monolithically have been developed [11-15].

In monolithic integration, several approaches to create regions with different material bandgaps on the same substrate have been presented in literature. The different material bandgaps are needed because active and passive devices require bandgap that is absorbing and nonabsorbing at the operating wavelength, respectively. One of such approaches is the etch-and-regrowth process. However, this method tends to sacrifice simplicity and yield and exacerbate the scattering and reflection losses at the growth interfaces. quantum-well intermixing (QWI) [16-20] is a relatively simple alternative which involves postgrowth manipulation of the bandgap of a quantum well, and it allows the active and passive devices to coexist on the same material layer without regrowth.

All the above-mentioned approaches are limited to lateral integration and require both the active and passive devices to share the same layer thicknesses and doping, which is not always desired. Active devices usually require smaller thickness for the light confinement layer and higher doping at the contact layer, such as those in lasers diode or in modulators. For passive devices like waveguides, such requirement is detrimental. In fact, larger waveguides have less propagation loss, lower fiber coupling loss and are relatively easier to fabricate. Lower doping also reduces the free carrier loss.

Most of the problems discussed above can be relieved by using vertical integration, in addition to planar integration. Vertical integration integrates devices vertically, and the devices on different vertical levels communicate through the vertical coupling of light. Hence, a vertical coupler, which comprises two vertically stacked waveguides capable of coupling light between each other, is essential as it provides the way to facilitate vertical integration. Because the devices and waveguides are constructed on different lateral planes, they are able to provide several useful advantages and solutions to the problem discussed previously, and these are summarized below.

(i) The devices can have different layer thicknesses. For example, one can have a thicker and wider waveguide core as the input waveguide to an active device, which has very thin and narrow core. The larger waveguide can improve the fiber coupling because it increases the modal overlap with the optical fiber.

(ii) It allows the flexibility in having different material bandgaps, doping concentrations, or refractive indexes in different layers. This can be done in just a single epitaxy growth.

(iii) It provides another dimension of photonic integration, which is the vertical integration.

Together with other planar integration techniques, they can lead to a 3-D high-density PIC.

Due to these advantages, the vertical coupler structure, being one form of spot-size converter or sometimes being denoted as asymmetric twin waveguides technology (ATG), has been studied in details [21-23] and utilized in diverse applications [24-43].

A common property of the $\mathrm{VC}$ is that it is a polarization-dependent device due to the polarizationdependent wave propagation in the ridge waveguides as well as the polarization-dependent coupling between them. This polarization sensitivity will transfer to the actual device. A polarization sensitive device may have limited applications in fiber-optic communication systems in which the polarization states of input optical signals to the photonic devices may change randomly as a function of time. Hence, this has imposed a need for a polarization-independent VC. On the other hand, there may be some situations in which a polarization-dependent coupler is useful. For example, in polarization-diversity detection, the VC may be used as a polarization splitter, in addition to serving as the low-loss coupler to the detector, as shown conceptually in Figure 1. In some cases, a variable polarization-dependent coupler may be used to compensate for the polarization dependence in the fiber coupling efficiency. This flexibility makes the vertical coupler a very compact and unique device, quite distinct from other polarization splitters based on other architectures or material platforms [44-49].

In this paper, we present our studies on the polarization dependence of the asymmetric-waveguides VC with a view to its application as an input-stage device with variable polarization dependence. Some new results are reported, but to be more comprehensive, we also review some of our previous results. As far as we know, the polarization dependence aspect of vertical couplers has not been reported before us, and it is only recently that some researchers have reported similar results $[50,51]$ that cited our works [52-54].

In the following sections, we will review five design variations from us, being

(i) polarization-independent (PI) coupler with the tapered transfer section designed around the region where the vertical coupler is almost polarization independent;

(ii) polarization-dependent coupler (PDC), or polarization mode splitter (PMS), designed to employ the polarization-dependent characteristic of the asymmetric waveguides vertical coupler; 
(iii) three-layer VC, a generalization of the two-layer case;

(iv) single-mesa vertical coupler, which could simplify the fabrication process of conventional double-mesa vertical coupler.

All these summarize our works done on the polarization dependence aspect of asymmetric-waveguides vertical couplers. The proposed concepts show the novelty of the works, and they will be emphasized. The device performances presented are the best effort of the authors, but by no means, the best achievable from the proposed concepts. Other simulation methods could also yield different or better optimized results, but it is not within the scope of the paper, and, thus, the paper does not pursue further into this.

There are also other material systems that could be employed in realizing the vertical coupler architecture. One example is the silicon-on-insulator (SOI), and it has been reported in [51, 55]. However, it is the scope of this paper to present the examples in InP-based compound semiconductor only; hence, other materials are not discussed despite their well-perceived potential. It is also noted that the wavelength dependence of vertical couplers could be of great interest, but it is beyond the coverage of this paper, as it requires more detail and extensive studies. They might form the future work of the author and would publish elsewhere.

In all simulations, the operating wavelength of the device is assumed to be $1.55 \mu \mathrm{m}$, and the three-dimensional (3D) semivectorial finite-difference beam-propagation method (FDBPM) is used [56]. All core materials are assumed to be InGaAsP with different compositions, and all cladding or spacer materials are assumed to be InP. The composition of various elements in InGaAsP, corresponding to the various refractive indexes can be estimated with Broberg's model [57].

\section{Design of Polarization-Independent Vertical Couplers}

The schematic of our VC is shown in Figure 2. The VC consists of two asymmetric waveguides one on top of another, separated by a thin spacer layer. The lower waveguide has a large uniform cross-section, while the upper waveguide is tapered and has a smaller core and higher refractive index than the underlying waveguide. For our illustration, we assume the substrate and the spacer material to be InP, and the waveguide core material to be InGaAsP. For typically application, the material structure of the upper waveguide is usually predetermined because it contains the actual integrated device. The designer has control only over the structure of the underlying passive waveguide.

For integration, the compactness of the $\mathrm{VC}$ is important. Hence, resonant coupling is the dominant mechanism being utilized in our design of the VC. Resonant coupling occurs between two asymmetric waveguides as long as they have the same effective index. However, practically the effective index of these waveguide is not known or controlled precisely. Therefore, the upper waveguide of an asymmetric waveguide VC is usually designed to be slowly tapered, with initial and final widths values enclosing the critical region for resonant

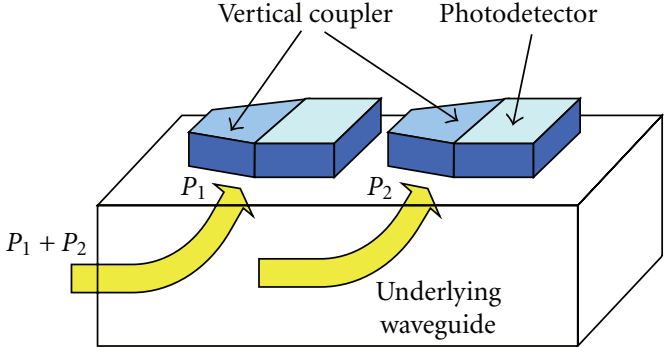

FIGURE 1: Illustration of polarization-diversity detection employing the vertical coupler polarization splitter. P: polarization.

coupling. A taper waveguide allows more tolerance to the deviation of the actual material indexes and the operating wavelength than a uniform waveguide. It is more effective to taper the upper waveguide, rather than the lower waveguide, because the upper waveguide effective index is more sensitive to width variations. Many tapering mechanisms have been discussed in literatures, but they are not within the scope of this paper, and, hence, they will not be discussed.

The effective indexes, $N_{\text {top }}$ of a tapered upper waveguide are a function of width only for the TE and TM polarizations, provided a given combination of core index and core thickness. This is illustrated in Figure 3. The waveguide birefringence ensures that the effective index is generally different for TE and TM polarizations, except at a specific width, which we denoted as the critical width, $W_{c}$, where they are equal. The waveguide birefringence is due to the material and structural asymmetry of a ridge waveguide that causes the TE and TM waves to see different material interfaces. This difference in interfaces will affect their mode distributions and subsequently their effective indexes. At the critical width, the waveguide is said to be polarization independent. For the lower waveguide, it is approximately polarization independent due to its relatively large and symmetric geometry. It has a single effective index value, $N_{\text {low }}$ and is represented by the horizontal line in Figure 3.

With our VC structure, resonant coupling occurs at the point on tapered waveguide, where $N_{\text {top }}=N_{\text {low }}$. We denote the width of the tapered upper waveguide at which this occurs as the resonant width, $W_{r}$. The resonant widths are generally different for TE and TM polarizations, which also mean that the resonant transfer occurs at different points along the taper. Thus, a VC is generally polarization sensitive. However, if the resonant widths coincide with the critical width $W_{r}=W_{c}$, then the coupling becomes relatively polarization insensitive. These behaviours form the basic principle for our design of VCs with arbitrary polarization dependence.

For a polarization independent VC, we design the coupled waveguides such that the TE and TM resonant widths are as close as possible to the critical width. For polarization mode splitter, on the other hand, we maximize the difference between the TE and TM resonant widths, which also tend to bring the resonant widths as far as possible from the critical width. Nevertheless, in either case, it is important to know the critical width. Therefore, in [54], we have plotted $W_{c}$ and its corresponding effective index $N_{c}$ 


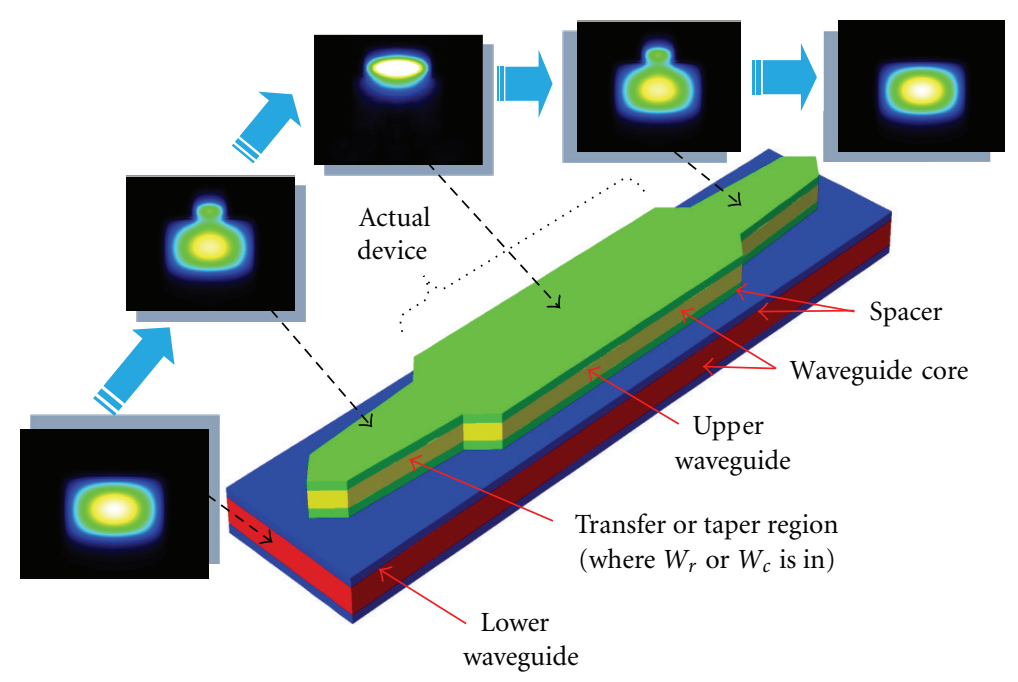

FIGURE 2: Schematic of two vertical couplers arranged in back-to-back configuration. Insets illustrate the transformation of the waveguide modes profile at the different sections of the device. (Not drawn to scale.)

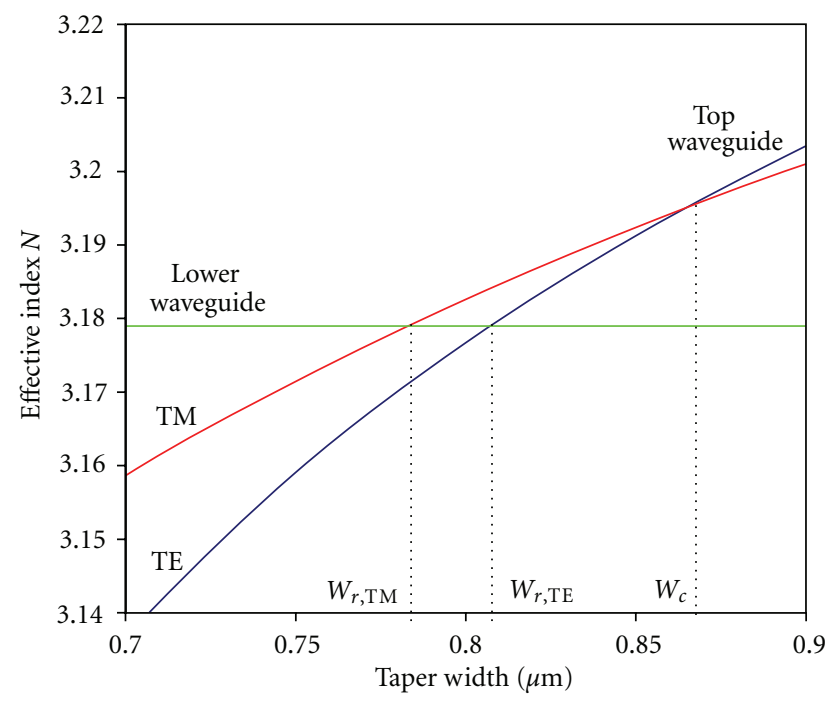

FIGURE 3: Illustration of the effective-index change for the interacting waveguides for TE and TM modes at various taper widths.

as a function of the waveguide core thickness and the core refractive index.

In the design of a polarization-independent VC, we need to ensure that $W_{r, \mathrm{TE}}=W_{r, \mathrm{TM}}$. As the freedom of deciding the top waveguide structure is generally determined by the optical device to which a VC is attached, [54] may be used to give critical width and effective index of the upper waveguide for any combination of core thickness and refractive index. The lower waveguide is then designed separately to match its effective index to $N_{c}$, the effective index of the upper waveguide at the critical width (i.e., matching the critical width and the resonant width). Finally, the design work is followed by the optimization of the taper width profile for the top waveguide, which yields the acceptable transfer efficiency.

As a representative example, we consider the case where the top waveguide is designed to be a passive device. As such, the top waveguide core is assumed to have a material index of 3.32 and a thickness of $0.7 \mu \mathrm{m}$. For this structure, the critical width is $1.85 \mu \mathrm{m}$. The lower waveguide has a core dimension of $4 \times 3 \mu \mathrm{m}^{2}$, and the core index is chosen to be 3.25 so that its effective index is $N_{\text {low }}=N_{c}$. For this exemplary design, the passive device is assumed to be $3.0 \mu \mathrm{m}$ in width. The transfer region (taper) is tapered from 1.40 to $2.00 \mu \mathrm{m}$ over a transfer length of $150 \mu \mathrm{m}$.

The simulation result is shown in Figure 4. The transfer efficiency is found to be greater than $90 \%$. The small discrepancy in transfer efficiency between the TE and TM polarizations is due to the residual birefringence accumulated over the transfer region. The power oscillations at the actual device region are due to mode beating between the upper and lower waveguides that are still weakly coupled. The oscillations may be reduced by downtapering the lower waveguide between the transfer region and the actual device to reduce its effective index further away from that of the upper waveguide.

\section{Design of Polarization Mode Splitters}

The VC can be designed as a PMS or filter that allows transfer of only one polarization between the lower waveguide and the upper waveguide. The main difference between the designs of PMS VC with the polarization-independent VC is the guiding principle of taper design. For the design of PMS, we maximize the difference between the resonant widths for the TE and TM modes in order to maximize the difference in their transfer efficiencies. This means that after the upper waveguide design (as defined by the core layer thickness and refractive index) is determined by the device application, the subsequent task is to design the lower waveguide to yield resonant widths (i.e., intercepting points) that are as far away from the critical width as possible. The taper waveguide is then designed such that the initial and final widths enclose only the resonant width of the 


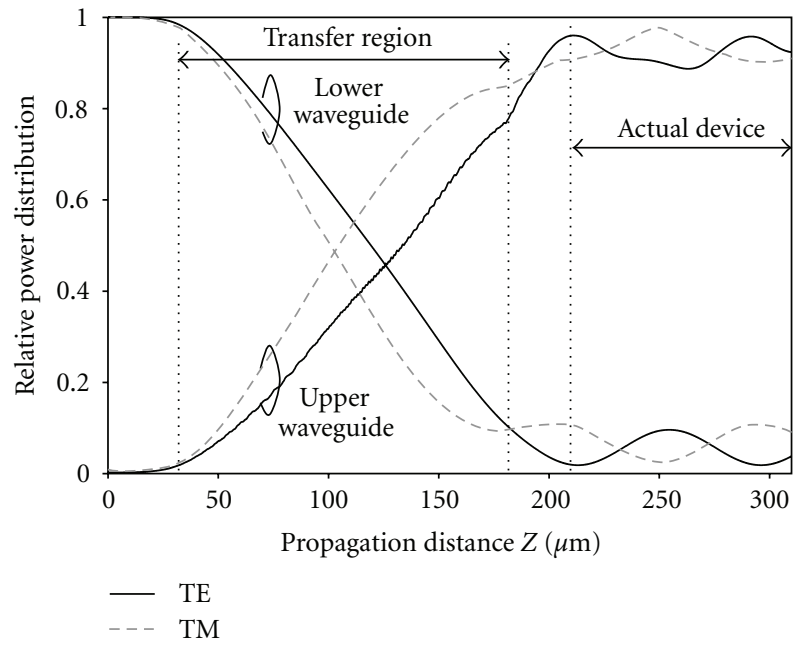

(a)

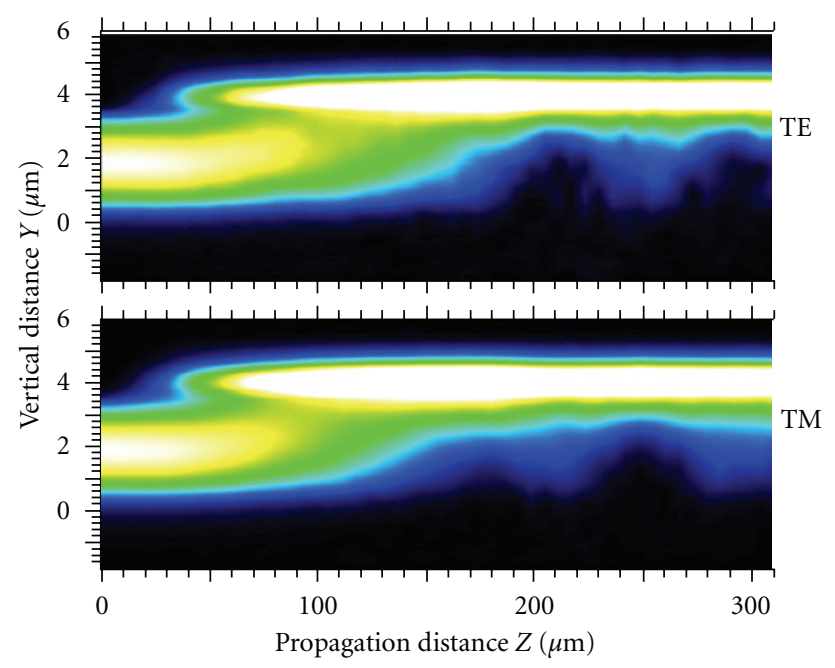

(b)

Figure 4: Transfer efficiency and transverse contour plots of the TE and TM polarization modes as a function of the propagation distance for an asymmetric-waveguides polarization-independent VC.

desired polarization. This thereby yields a high contrast ratio between the two polarizations in the upper waveguide.

Depending on the relative refractive indexes of the upper and lower waveguides, the resonant widths can occur to the right or to the left of the critical width. The latter case is shown in Figure 5, again based on our representative example except that the lower waveguide now has a core index of 3.20. In the figure, the dotted curves represent the effective index of the uncoupled lower and upper waveguides, while the bold solid curves give the effective indexes of the supermodes (for TE and TM) for the coupled structure, showing how the supermodes evolve from the lower to the upper waveguide as the upper waveguide taper width is increased. Note that there exists a region, denoted as the polarization-dependent (PD) region, near the first resonant width where one of the polarizations has started to transfer light onto the upper waveguide, while there is not yet any

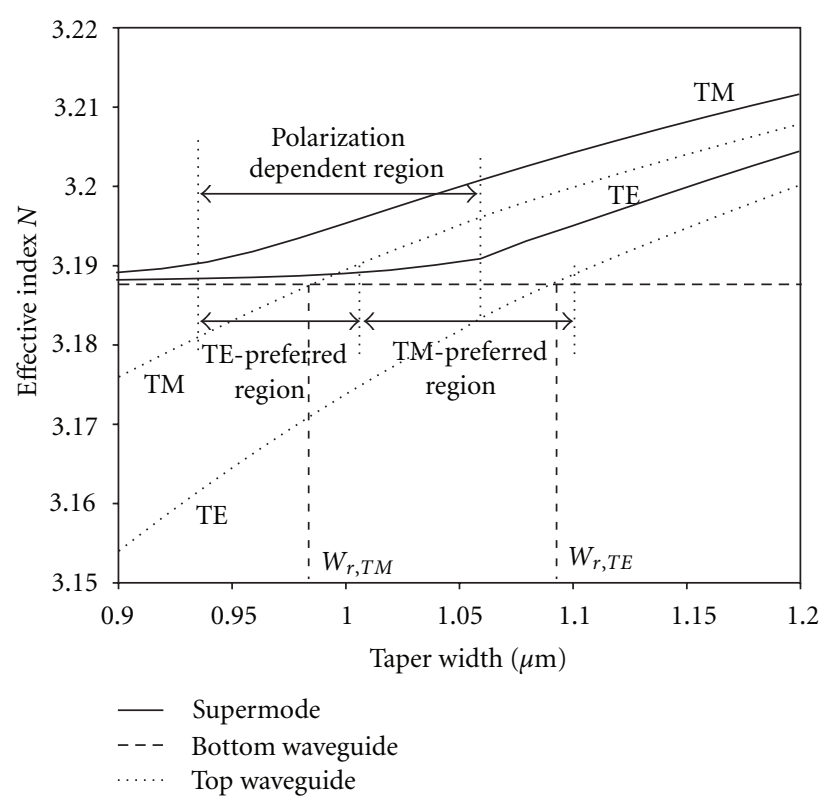

FIGURE 5: Effective indexes as a function of the taper widths for the confined TE and TM supermodes (bold curves) of the VC and the individual (uncoupled) waveguides (dotted curves). The parameters are discussed in the text.

light transfer for the other polarization. It is found that the farther the resonant widths are from the critical width and the steeper the effective index curves are, the wider this $\mathrm{PD}$ region will be. The wider the $\mathrm{PD}$ region, the better the contrast ratio will be between the two polarizations.

The design of the case where the resonant widths are to the right of the critical width tends to have poorer performance. Note that this is because the $\mathrm{PD}$ region in this case is narrower due to the smaller slope of the effective index curve. The advantage in this region, however, is that the resonant widths are somewhat larger, and the taper is easier to fabricate.

In our exemplary design, the VC structure is the same as that used for Figure 5, where the upper waveguide core has a refractive index of 3.32 and a thickness of $0.7 \mu \mathrm{m}$, and the bottom waveguide has a refractive index of 3.20 corresponding to an effective index of 3.189. The resonant widths are seen to be $0.985 \mu \mathrm{m}$ (TM) and $1.09 \mu \mathrm{m}$ (TE). The transfer region for the TM-preferred case is, therefore, tapered from 0.90 to $1.00 \mu \mathrm{m}$. For the TE-preferred case, the transfer region is tapered from 1.00 to $1.10 \mu \mathrm{m}$. The taper lengths are $150 \mu \mathrm{m}$. The simulation results are presented in Figure 6 . Note that the contrast ratio for the TE-preferred case is about $6: 1$ (TE:TM =95\%:16\%), and for the TMpreferred case is about $5: 1$.

The type of device application dictates the upper waveguide structure, which in turn determines the lower waveguide refractive index and the initial and final widths of the taper waveguide. Comparison shows that the active device, because the core index is generally higher, has a smaller critical width and, hence, requires a smaller taper with very small width differentials. The resulting transfer ratios indicated for the PMSs are by no means the best that 


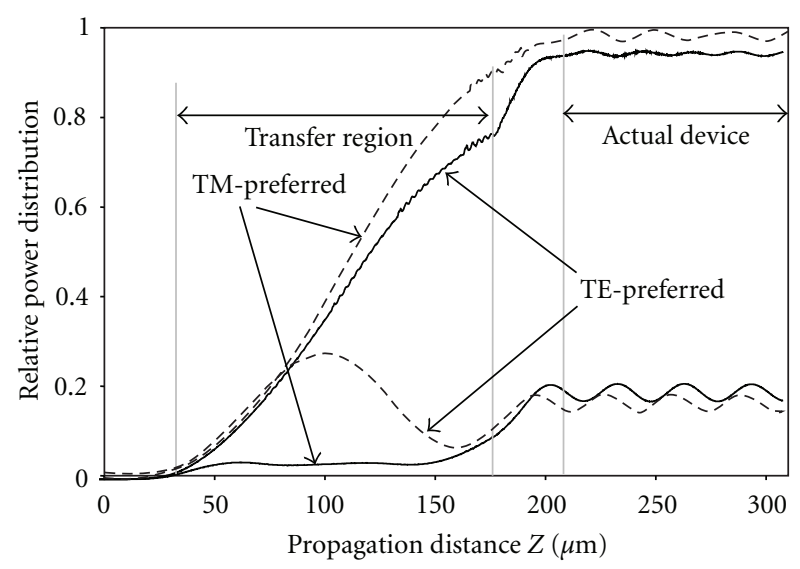

(a)

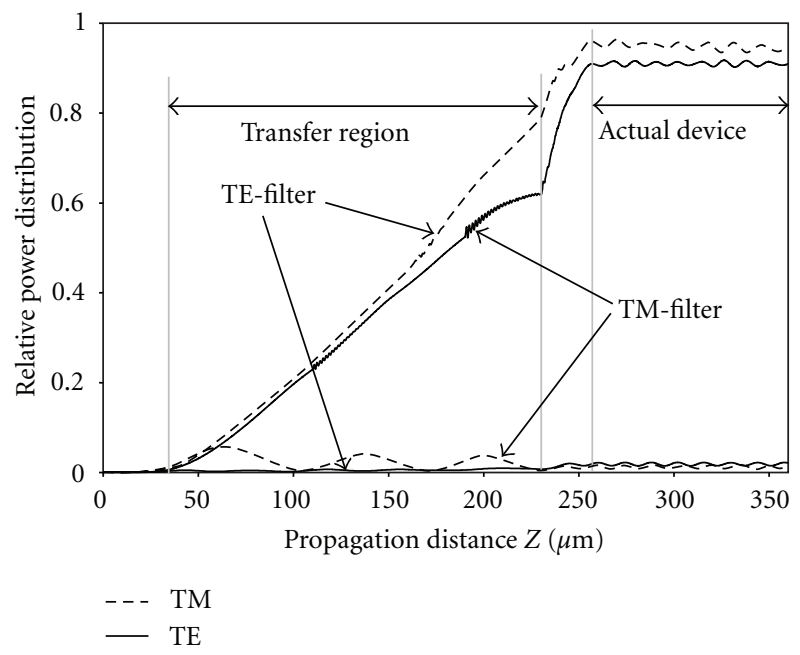

(b)

FIgure 6: Transfer efficiency for the TE and TM polarization modes as a function of the propagation distance for an asymmetricwaveguide PMS as an input stage of (a) a passive device and (b) an active device.

can be achieved but rather are constrained to some extent by the practical limits we have imposed on the smallest taper width.

\section{Demonstration of Single-Mesa Vertical Coupler}

The conventional fabrication process of these vertical couplers is not straight forward. The multiple mesas require multiple photolithography and etching steps, and thus resulting in a nonplanar structure. Planarization and etch back are usually required to ease the critical alignment of the upper waveguide with respect to the lower mesa. Hence, it is desirable to minimize the number of process steps. In this section, we propose a single-etch/mesa vertical coupler based on an underlying slab waveguide and a top ridge waveguide [58]. The structure of single-mesa VC is quite different from the double-mesa VC presented in the previous section, and, hence, their comparison is inessential.

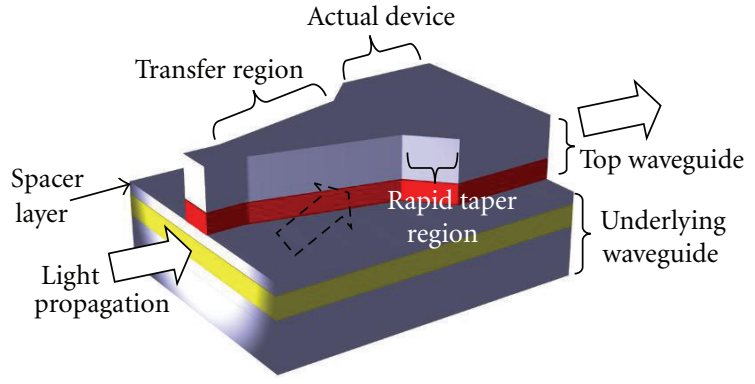

Figure 7: Schematic of single-mesa vertical coupler.

The underlying waveguide consists of a slab material, in which the weak lateral guiding is given by the loading effect obtained from the top ridge waveguide. Hence, no additional fabrication steps are required to define the underlying waveguide, and the critical alignment between the two vertically stacked waveguides can be avoided. The larger, weakly guiding underlying waveguide acts as the input coupling waveguide while the actual device is on the top waveguide. Due to the larger and weakly guiding nature of the underlying input waveguide, it poses the potential of improving the fiber-coupling loss.

The schematic of the single-mesa vertical coupler is shown in Figure 7. The two waveguides are separated by a spacer layer. The top/upper waveguide consists of a uniform section, which provides the loading effect for the bottom/lower input waveguide, followed by a linear slow taper section that acts as the light transfer region to the actual device. This slow taper section exists to relax the strict fabrication tolerance for the vertical coupling region. After that, it is uptapered rapidly over a distance of $30 \mu \mathrm{m}$ to the width of the actual device, which is assumed to be $2 \mu \mathrm{m}$. This rapid taper section serves as a transition between the transfer region and the actual device, and it effectively cuts off further coupling oscillation between top and bottom waveguides. The length could be shorter, but the abrupt change of effective index seen by the light might cause reflection. $30 \mu \mathrm{m}$ is found to have a smooth transition. To show its capability to be integrated with other photonic device, we further demonstrate an integrated device between the single-mesa VC and an MMI.

The material structure of the actual device is assumed to be predetermined by application, and, in our study, the device waveguide core is assumed to have a material index of 3.47 , a thickness of $0.6 \mu \mathrm{m}$, and a width of $2 \mu \mathrm{m}$. The underlying waveguide core is assumed to have an index of 3.40. The spacer, claddings, and substrate are all assumed to be InP with an index of 3.17. The remaining variable parameters are the lower waveguide core thickness, the spacer thickness, the upper waveguide initial and final taper widths, and the taper length. For the fiber coupling consideration, we assume a standard single-mode fiber with a mode-field diameter of $10 \mu \mathrm{m}$. From the simulation, as we shall see, an improvement of $8 \mathrm{~dB}$ over direct coupling into the actual device is obtained with the single-mesa VC.

In the single-mesa $\mathrm{VC}$, the bottom rib waveguide is responsible for the improvement of fiber coupling. The 
thickness of the bottom waveguide core plays an important role in deciding the mode profile of the input mode, which in turn affects the fiber coupling. From our study [58], for a spacer thickness of $0.3 \mu \mathrm{m}$, it is found that the optimum core thickness is $0.7 \mu \mathrm{m}$, which gives a coupling loss of $6.5 \mathrm{~dB}$. This is an improvement of $8.5 \mathrm{~dB}$ compared to direct coupling into the top waveguide. A reasonably thick spacer layer, such as in this case of $0.3 \mu \mathrm{m}$, is chosen to ease the control of subsequent selective etching process. Higher spacer thickness is not preferred so that the overall device height is reduced (for stronger light transfer and easier epi growth). Thick spacer layer also tends to weaken the lateral light confinement at the underlying waveguide. This reduces the fiber coupling as the waveguide mode becomes more asymmetric.

The loading effect for the bottom rib waveguide is supplied by the uniform section of the top waveguide. The waveguide width must not be too wide to shift the guiding mode to the upper waveguide or too narrow to make fabrication difficult. With the spacer thickness of $0.3 \mu \mathrm{m}$ and bottom core thickness of $0.7 \mu \mathrm{m}, 1 \mu \mathrm{m}$ is found to be the optimum width that gives the lowest coupling loss.

As similar to other VCs in the paper, a single-mesa vertical coupler transfers light to the top waveguide through resonant coupling. The effective index of (i) underlying rib waveguide at the present of the uniform region of the tapered top waveguide, and (ii) top waveguide alone as its width is varied along the taper is plotted in Figure 8. The effective indices are polarization dependent due to the asymmetric waveguide structure; hence, there are two resonant widths, one for TE polarization and another for TM polarization. This is quite a different phenomenon from previous VCs. However, we find that if the transfer region (slow taper region) encompasses both resonant widths and the resonant widths are close to $W_{c}$, then the polarization dependence in transfer efficiency is negligible. For our design in Figure 8, critical width, TE and TM resonant widths are close to each other, which is around $1.2 \mu \mathrm{m}$.

With the initial taper width fixed at $1 \mu \mathrm{m}$ for reasons given previously, the final width of the transfer region is varied to maximize the transfer efficiency. The transfer efficiencies as a function of the final taper widths are calculated. The transfer length is fixed at $150 \mu \mathrm{m}$. It is found that at the final taper width of $1.3 \mu \mathrm{m}$, the transfer efficiencies for both polarizations are more than $80 \%$ and are almost equal. This width, together with the initial taper width, encompasses the resonant widths and the critical width of around $1.2 \mu \mathrm{m}$. We also found that the transfer efficiency is higher and less sensitive to the final width for larger taper length due to the adiabatic coupling that occurs in a longer transfer region. Hence, to achieve a more compact and robust device, the transfer length of $150 \mu \mathrm{m}$ is chosen in our design.

The 3D-FDBPM simulation results for the aboveoptimized results are shown in Figure 9. At the initial region, the overlap integral with the eigenmode of the top waveguide is not zero because the rib waveguide is quite heavily loaded, such that the input mode fields are extended slightly into the top waveguide. It can be seen that the TM polarization, which has the dominant electric field in the vertical direction, couples more strongly and rapidly to the upper waveguide

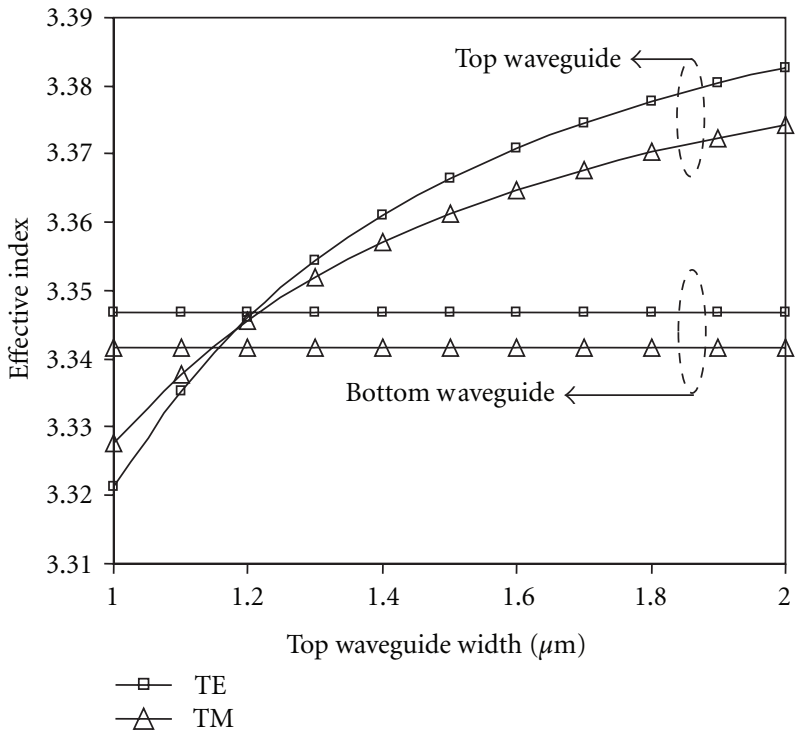

Figure 8: Effective index of the upper and the lower waveguides, for TE and TM polarizations, when the upper waveguide width is changed. The top waveguide width has a core index of 3.50 and thickness of $0.6 \mu \mathrm{m}$. The lower core material index is assumed to be 3.42 , with a thickness of $0.7 \mu \mathrm{m}$. The spacer thickness is $0.3 \mu \mathrm{m}$.

than the TE polarization, possibly due to the larger overlap between the two waveguide modes. For TM polarization alone, shorter transfer region could be expected, but in order to minimize the device polarization sensitivity, longer transfer region is designed instead. In the actual device region, some back coupling is observed because of the mode beating between the two weakly coupled waveguides. Lower beating could be achieved by further optimization of the actual device layer, which is beyond the scope of this paper. It is also found that the device performance is relatively broadband by having a bandwidth of $10 \mathrm{~nm}$, similar to our previous reported results [54].

The fabrication of the single-mesa structure is much simplified than the conventional double-mesa VC, where only one round of ridge waveguide fabrication process is carried out. The epitaxy wafers are purchased commercially with the layer structure as described previously. The epitaxy samples are first deposited by Unaxis Nextral D200 plasma-enhanced chemical vapor deposition (PECVD) $\mathrm{SiO}_{2}$, which acts as the etch mask for subsequent waveguide etching. Then, the waveguide patterns are transfer through photolithography process using an electron-beam-written photomask for better dimension fidelity. The mask aligner used is the Suss MJB4. Oxford Plasmalab 80Plus reactive ion etching (RIE) is then used to etch the $\mathrm{SiO}_{2}$ etch mask, and the exposed InP compound underneath is etched by $\mathrm{Cl}_{2} / \mathrm{CH}_{4} / \mathrm{H}_{2}$ plasma recipe developed previously [59] with an Oxford Plasmalab 100 inductively coupled plasma (ICP) system.

In the fabrication, series of single-mesa VCs with slight deviation $( \pm 10 \%)$ in the waveguide dimensions are fabricated. Each one is fabricated side by side with (i) a rib waveguide, that is, the underlying waveguide with the presence of a 


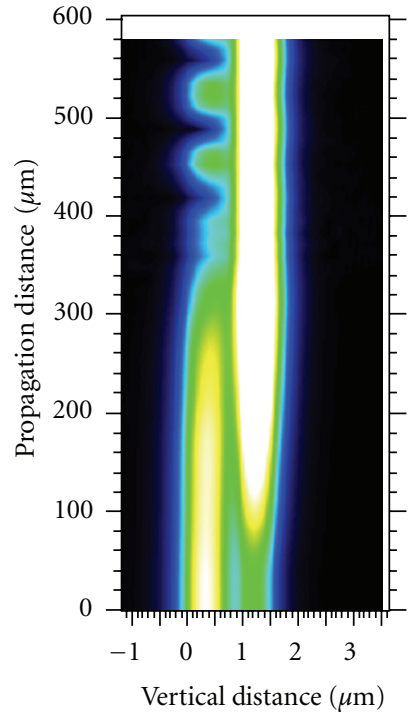

(a)

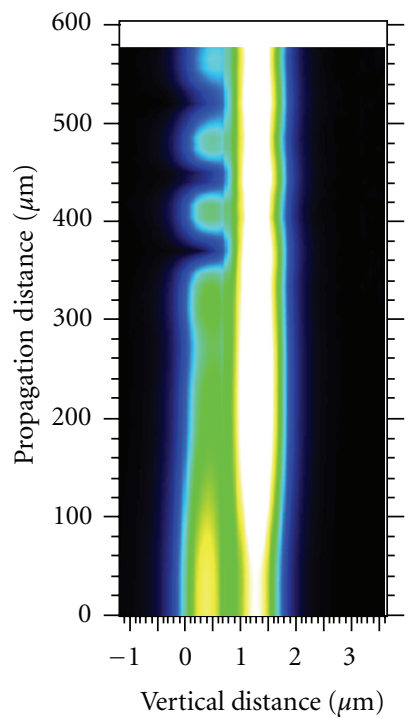

(b)
Figure 9: Contour plot showing the light transfer to the top waveguide $(Y>1 \mu \mathrm{m})$, for TE and TM polarizations along the propagation distance.

uniform $1 \mu \mathrm{m}$ top waveguide and (ii) the integrated $\mathrm{VC}$ and $1 \times 2 \mathrm{MMI}$ device. This is to facilitate testing and verification of the VC and the integrated device. Although variation is incorporated into the device dimension, in the actual fabrication, the dimension of the devices could still deviate from the designed values due to the process resolution and environment. The deviation of dimension is probably not critical for the MMI, as MMI is a robust device and allows larger fabrication tolerances. However, for the vertical coupler, this is not the case. Nevertheless, by tuning the operating wavelength, the intersection of the effective index curves can be tuned, and, therefore, the phase matching condition could be adjusted to fit within the taper region.

Experimentally, it is found that at the operating wavelength of about $1530 \mathrm{~nm}$, the performance of the vertical coupler is optimum. The mode profile of the output from the rib waveguide (i.e., the top waveguide is uniform with a width of $1 \mu \mathrm{m}$ ) is shown in Figure 10(a), where we can see that the mode profile is relatively larger. The mode profile shows a tail at the top, which is a typical mode shape for a rib waveguide. At the output of the tapered top waveguide, the mode profile is smaller and dimmer, as shown in Figure 10(b). In one of the devices, insertion losses of about $13 \mathrm{~dB}$ and $21 \mathrm{~dB}$ for the underlying waveguide and vertical coupler, respectively, are obtained. The chip/total device lengths are about $2 \mathrm{~mm}$.

From our measurement of the propagation loss for the underlying waveguide with Fabry-Perot method [60], the propagation loss is only about $0.4 \mathrm{~dB} / \mathrm{cm}$, probably because in the rib structure, there is much less light scattering at the waveguide sidewall. If the propagation loss at the short underlying waveguide is negligible, then the fiber coupling loss is about $13 \mathrm{~dB}$, which includes the facet reflection loss. With this, we can estimate that the transfer loss and the

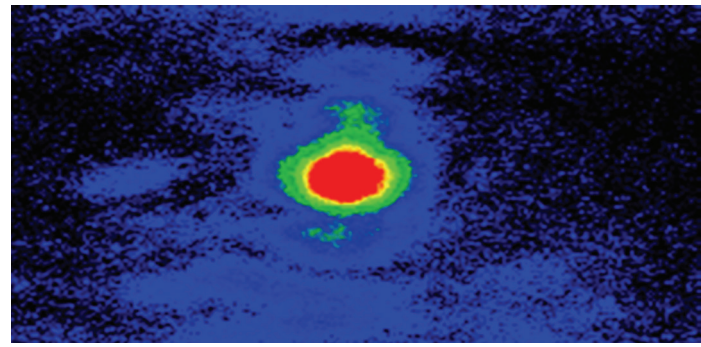

(a)

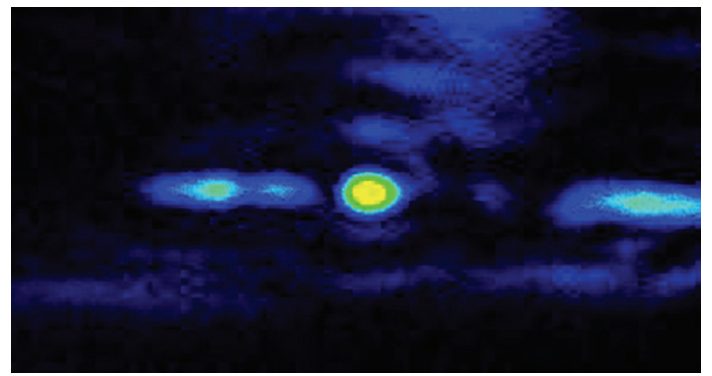

(b)

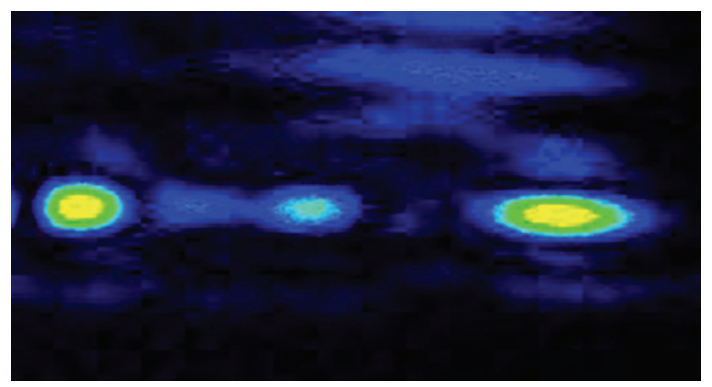

(c)

FigURE 10: Measured output mode profiles from (a) underlying rib waveguide, (b) single-mesa vertical coupler, and (c) integrated device of single-mesa VC and $1 \times 2 \mathrm{MMI}$.

propagation loss in the top waveguide is about $8 \mathrm{~dB}$, and they are inseparable. Based on these data, we can also estimate that the minimum transfer efficiency we could achieve is $16 \%$.

We also demonstrate the integration of the single-mesa VC with a $1 \times 2$ MMI. The MMI body is $8 \mu \mathrm{m}$ in width and $69 \mu \mathrm{m}$ in length. The input port is placed at the centre of the MMI, while the output ports are placed symmetrically about the centre of the MMI, with a $4 \mu \mathrm{m}$ separation amongst them. With this configuration, symmetric restricted interference is induced in the MMI [61], giving a compact MMI structure. In Figure 10(c), we show the output mode profiles from the $1 \times 2$ MMI. The imbalance in the mode intensities is due to the slight imperfection of the cleaving at the output facet. The output powers at the two ports give a splitting ratio of almost 50:50 and an overall device insertion loss (VC + MMI) of about $19 \mathrm{~dB}$.

\section{Conclusion}

This paper presented a review of systematic approach to the design of compact VC mode-size converters with variable 
polarization dependence. The design principle is based on resonant coupling between the top-tapered waveguide and the underlying uniform and large waveguide. As a PI coupler, the VC is designed so that resonant transfer occurs near the critical width where the upper waveguide is nonbirefringent, and the transfer efficiency is greater than $90 \%$ for both TE and TM polarizations over a length as small as $150 \mu \mathrm{m}$. More generally, the $\mathrm{VC}$ is polarization dependent because the resonant widths and the critical width do not coincide, and, by varying the spacing between them, we can design a polarization-dependent coupler with an arbitrary ratio between the TE and TM transfer efficiencies. In the limiting case of a polarization-mode splitter, equally compact polarization splitters with a contrast ratio of $20 \mathrm{~dB}$ can be achieved. We further demonstrate experimentally the single-mesa VC, which simplifies the fabrication process and potentially could improve the process yield, and its integration with a multimode interferometer (MMI). The versatility makes the VC a compact and useful inputstage device that not only maximizes input/output coupling efficiency to small active devices but also provides a degree of polarization control before the actual device.

\section{Acknowledgments}

The author would also like to express his sincere appreciation to the guidance of late Assoc. Prof Mee-Koy Chin from Nanyang Technological University Singapore. The author also gratefully acknowledges the support of A*STAR Singapore through the postgraduate and the overseas postdoctoral sponsorships.

\section{References}

[1] K. Kawano, M. Kohtoku, M. Wada, H. Okamoto, Y. Itaya, and M. Naganuma, "Design of a spotsize-converter-integrated laser diode (SS-LD) with a lateral taper, thin-film core and ridge in the $1.3 \mu \mathrm{m}$-wavelength region based on the 3-D BPM," IEEE Journal on Selected Topics in Quantum Electronics, vol. 2, no. 2, pp. 348-354, 1996.

[2] Y. Inaba, M. Kito, T. Nishikawa, M. Ishino, and Y. Matsui, "Multiquantum-well lasers with tapered active stripe for direct coupling to single-mode fiber," IEEE Photonics Technology Letters, vol. 9, no. 6, pp. 722-724, 1997.

[3] V. Vusirikala, S. S. Saini, R. E. Bartolo et al., “ $1.55-\mu \mathrm{m}$ InGaAsP-InP laser arrays with integrated-mode expanders fabricated using a single epitaxial growth," IEEE Journal on Selected Topics in Quantum Electronics, vol. 3, no. 6, pp. 13321343, 1997.

[4] R. Y. Fang, D. Bertone, M. Melinga, I. Montrosset, G. Oliveti, and R. Paoletti, "Low-cost $1.55^{-} \mathrm{m}$ InGaAsP-InP spot-sizeconverter (SSC) laser with conventional active layers," IEEE Photonics Technology Letters, vol. 9, pp. 1084-1086, 1997.

[5] K. Kawano, M. Kohtoku, H. Okamoto, Y. Itaya, and M. Naganuma, "Coupling and conversion characteristics of spotsize-converter integrated laser diodes," IEEE Journal on Selected Topics in Quantum Electronics, vol. 3, no. 6, pp. 13511359, 1997.

[6] Y. Kawamura, K. Wakita, Y. Yoshikuni, Y. Itaya, and H. Asahi, "Monolithic integration of a DFB laser and an MQW optical modulator in the $1.5^{-} \mathrm{m}$ wavelength range," IEEE Journal of Quantum Electronics, vol. QE-23, no. 6, pp. 915-918, 1987.

[7] H. Soda, M. Furutsu, K. Sato et al., "High-power and highspeed semi-insulating BH structure monolithic electroabsorption modulator/DFB laser light source," Electronics Letters, vol. 26, no. 1, pp. 9-10, 1990.

[8] B. Mason, G. A. Fish, S. P. DenBaars, and L. A. Coldren, "Widely tunable sampled grating DBR laser with integrated electroabsorption modulator," IEEE Photonics Technology Letters, vol. 11, no. 6, pp. 638-640, 1999.

[9] J. E. Johnson, L. J. P. Ketelsen, S. K. Sputz et al., "Monolithically integrated semiconductor optical amplifier and electroabsorption modulator with dual-waveguide spot-size converter input," IEEE Journal on Selected Topics in Quantum Electronics, vol. 6, no. 1, pp. 19-25, 2000.

[10] B. Mason, J. Barton, G. A. Fish, L. A. Coldren, and S. P. DenBaars, "Design of sampled grating DBR lasers with integrated semiconductor optical amplifiers," IEEE Photonics Technology Letters, vol. 14, pp. 1457-1459, 2002.

[11] M. G. Young, U. Koren, B. I. Miller et al., " $16 \times 1$ wavelength division multiplexer with integrated distributed Bragg reflector lasers and electroabsorption modulators," IEEE Photonics Technology Letters, vol. 5, no. 8, pp. 908-910, 1993.

[12] K. Kudo, K. Yashiki, T. Sasaki et al., " $1.55 \mu \mathrm{m}$ wavelengthselectable microarray DFB-LD's with monolithically integrated MMI combiner, SOA, and EA-modulator," IEEE Photonics Technology Letters, vol. 12, no. 3, pp. 242-244, 2000.

[13] R. Nagarajan, C. H. Joyner, R. P. Schneider Jr. et al., "Largescale photonic integrated circuits," IEEE Journal on Selected Topics in Quantum Electronics, vol. 11, no. 1, pp. 50-62, 2005.

[14] R. Nagarajan, M. Kato, V. G. Dominic et al., "400 Gbit/s (10 channel $\times 40 \mathrm{Gbit} / \mathrm{s})$ DWDM photonic integrated circuits," Electronics Letters, vol. 41, no. 6, pp. 347-349, 2005.

[15] R. Nagarajan, M. Kato, J. Pleumeekers et al., "Single-chip 40channel InP transmitter photonic integrated circuit capable of aggregate data rate of 1.6 Tbit/s," Electronics Letters, vol. 42, no. 13, pp. 771-773, 2006.

[16] D. Hofstetter, B. Maisenhölder, and H. P. Zappe, "Quantumwell intermixing for fabrication of lasers and photonic integrated circuits," IEEE Journal on Selected Topics in Quantum Electronics, vol. 4, no. 4, pp. 794-802, 1998.

[17] S. D. McDougall, O. P. Kowalski, C. J. Hamilton et al., "Monolithic integration via a universal damage enhanced quantumwell intermixing technique," IEEE Journal on Selected Topics in Quantum Electronics, vol. 4, no. 4, pp. 636-646, 1998.

[18] H. S. Djie, T. Mei, J. Arokiaraj et al., "Experimental and theoretical analysis of argon plasma-enhanced quantum-well intermixing," IEEE Journal of Quantum Electronics, vol. 40, no. 2, pp. 166-174, 2004.

[19] J. P. Reithmaier and A. Forchel, "Focused ion-beam implantation induced thermal quantum-well intermixing for monolithic optoelectronic device integration," IEEE Journal on Selected Topics in Quantum Electronics, vol. 4, no. 4, pp. 595605, 1998.

[20] H. S. Kim, J. W. Park, D. K. Oh, K. R. Oh, S. J. Kim, and I. H. Choi, "Quantum well intermixing of $\operatorname{In}_{1-x} \mathrm{Ga}_{x} \mathrm{As} / \mathrm{InP}$ and $\mathrm{In}_{1-x} \mathrm{Ga}_{x} \mathrm{As} / \mathrm{In}_{1-x} \mathrm{Ga}_{x} \mathrm{As}_{1-y} \mathrm{P}_{y}$ multiple-quantum-well structures by using the impurity-free vacancy diffusion technique," Semiconductor Science and Technology, vol. 15, no. 10, pp. 1005-1009, 2000.

[21] F. Xia, V. M. Menon, and S. R. Forrest, "Photonic integration using asymmetric twin-waveguide (ATG) technology: part I-concepts and theory," IEEE Journal on Selected Topics in Quantum Electronics, vol. 11, no. 1, pp. 17-29, 2005. 
[22] F. Xia, V. M. Menon, and S. R. Forrest, "Photonic integration using asymmetric twin-waveguide (ATG) technology: part II-devices," IEEE Journal on Selected Topics in Quantum Electronics, vol. 11, no. 1, pp. 30-42, 2005.

[23] X. Sun, H. C. Liu, and A. Yariv, "Adiabaticity criterion and the shortest adiabatic mode transformer in a coupled-waveguide system," Optics Letters, vol. 34, no. 3, pp. 280-282, 2009.

[24] L. Hou, Z. Ren, Y. Shu, and S. Yu, "Angled-facet spotsize-converter integrated semiconductor optical amplifiers using asymmetric twin waveguide technology," IEEE Photonics Technology Letters, vol. 20, no. 8, pp. 563-565, 2008.

[25] Y. D. Chung, J. S. Sim, S. B. Kim, J. Kim, and S. W. Ryu, "Fabrication and characterization of a spot-size converterintegrated $1.3 \nu \mathrm{m}$ FP laser diode," Semiconductor Science and Technology, vol. 21, no. 6, pp. 790-793, 2006.

[26] H. Ma, S. Chen, X. Yi, and G. Zhu, " $1.55 \mu \mathrm{m}$ spot-size converter integrated polarization-insensitive quantum-well semiconductor optical amplifier with tensile-strained barriers," Semiconductor Science and Technology, vol. 19, no. 7, pp. 846-850, 2004.

[27] K. T. Shiu, S. S. Agashe, and S. R. Forrest, "A simple monolithically integrated optical receiver consisting of an optical preamplifier and a p-i-n photodiode," IEEE Photonics Technology Letters, vol. 18, no. 8, pp. 956-958, 2006.

[28] Z. J. Cheng, Z. L. Peng, K. X. Chen, H. P. Chan, C. X. Yu, and P. L. Chu, "Polymer-waveguide-based vertical coupler," Optics Communications, vol. 260, no. 2, pp. 511-513, 2006.

[29] S. S. Agashe, S. Datta, F. Xia, and S. R. Forrest, "A monolithically integrated long-wavelength balanced photodiode using asymmetric twin-waveguide technology," IEEE Photonics Technology Letters, vol. 16, no. 1, pp. 236-238, 2004.

[30] F. Z. Lin, Y. J. Chiu, S. A. Tsai, and T. H. Wu, "Laterally tapered undercut active waveguide fabricated by simple wet etching method for vertical waveguide directional coupler," Optics Express, vol. 16, no. 11, pp. 7588-7594, 2008.

[31] K. T. Shiu, S. S. Agashe, and S. R. Forrest, "An InP-based monolithically integrated reconfigurable optical add-drop multiplexer," IEEE Photonics Technology Letters, vol. 19, no. 19, pp. 1445-1447, 2007.

[32] Y. Inaba, M. Kito, T. Nishikawa, M. Ishino, and Y. Matsui, "Multiquantum-well lasers with tapered active stripe for direct coupling to single-mode fiber," IEEE Photonics Technology Letters, vol. 9, no. 6, pp. 722-724, 1997.

[33] V. Vusirikala, S. S. Saini, R. E. Bartolo et al., " $1.55 \mu \mathrm{m}$ InGaAsP-InP laser arrays with integrated-mode expanders fabricated using a single epitaxial growth," IEEE Journal on Selected Topics in Quantum Electronics, vol. 3, no. 6, pp. 13321343, 1997.

[34] R. Y. Fang, D. Bertone, M. Melinga, I. Montrosset, G. Oliveti, and R. Paoletti, "Low-cost $1.55^{-} \mathrm{m}$ InGaAsP-InP spot-sizeconverter (SSC) laser with conventional active layers," IEEE Photonics Technology Letters, vol. 9, pp. 1084-1086, 1997.

[35] K. Kawano, M. Kohtoku, H. Okamoto, Y. Itaya, and M. Naganuma, "Coupling and conversion characteristics of spotsize-converter integrated laser diodes," IEEE Journal on Selected Topics in Quantum Electronics, vol. 3, no. 6, pp. 13511359, 1997.

[36] B. Mersali, A. Ramdane, and A. Carenco, "Optical-mode transformer: a III-V circuit integration enabler," IEEE Journal on Selected Topics in Quantum Electronics, vol. 3, no. 6, pp. 1321-1331, 1997.

[37] N. Yoshimoto, K. Kawano, H. Takeuchi, S. Kondo, and Y. Noguchi, "Highly efficient coupling semiconductor spot-size converter with an InP/InAlAs multiple-quantum-well core," Applied Optics, vol. 34, no. 6, pp. 1007-1014, 1995.

[38] B. Mersali, H. J. Bruckner, M. Feuillade, S. Sainson, A. Ougazzaden, and A. Carenco, "Theoretical and experimental studies of a spot-size transformer with integrated waveguide for polarization insensitive optical amplifiers," Journal of Lightwave Technology, vol. 13, no. 9, pp. 1865-1872, 1995.

[39] B. Liu, A. H. Shakouri, P. Abraham, Y. J. Chiu, S. Zhang, and J. E. Bowers, "Fused InP-GaAs vertical coupler filters," IEEE Photonics Technology Letters, vol. 11, no. 1, pp. 93-95, 1999.

[40] C. Wu, C. Rolland, F. Shepherd et al., "InGaAsP/InP vertical directional coupler filter with optimally designed wavelength tunability," IEEE Photonics Technology Letters, vol. 5, no. 4, pp. 457-459, 1993.

[41] R. C. Alferness, L. L. Buhl, U. Koren et al., "Broadly tunable InGaAsP/InP buried rib waveguide vertical coupler filter," Applied Physics Letters, vol. 60, no. 8, pp. 980-982, 1992.

[42] M. Raburn, B. Liu, K. Rauscher, Y. Okuno, N. Dagli, and J. E. Bowers, "3-D photonic circuit technology," IEEE Journal on Selected Topics in Quantum Electronics, vol. 8, no. 4, pp. 935942, 2002.

[43] B. Liu, A. Shakouri, P. Abraham, and J. E. Bowers, "Optical add/drop multiplexers based on X-crossing vertical coupler filters," IEEE Photonics Technology Letters, vol. 12, no. 4, pp. 410-412, 2000.

[44] M. Hotta and M. Geshiro, "Novel optical polarization splitter using a dimensionally tapered velocity coupler," IEICE Transactions on Electronics, vol. E77-C, no. 11, pp. 1722-1725, 1994.

[45] J. J. G. M. van der Tol, J. W. Pedersen, E. G. Metaal, J. J. W. Van Gaalen, Y. S. Oei, and F. H. Groen, "A short polarization splitter without metal overlays on InGaAsP-InP," IEEE Photonics Technology Letters, vol. 9, no. 2, pp. 209-211, 1997.

[46] L. B. Soldano, A. H. de Vreede, M. K. Smit, B. H. Verbeek, E. G. Metaal, and F. H. Groen, "Mach-Zehnder interferometer polarization splitter in InGaAsP/InP," IEEE Photonics Technology Letters, vol. 6, no. 3, pp. 402-405, 1994.

[47] N. Goto and G. L. Yip, "TE-TM mode splitter in LiNbO3 by proton exchange and Ti diffusion," Journal of Lightwave Technology, vol. 7, no. 10, pp. 1567-1574, 1989.

[48] P. Albrecht, M. Hamacher, H. Heidrich, D. Hoffmann, H. P. Nolting, and C. M. Weinert, "TE/TM mode splitters on InGaAsP/InP," IEEE Photonics Technology Letters, vol. 2, no. 2, pp. 114-115, 1990.

[49] S. M. Garner, V. Chuyanov, S. S. Lee, A. Chen, W. H. Steier, and L. R. Dalton, "Vertically integrated waveguide polarization splitters using polymers," IEEE Photonics Technology Letters, vol. 11, no. 7, pp. 842-844, 1999.

[50] M. Galarza, D. Van Thourhout, R. Baets, and M. Lopez-Amo, "Compact and highly-efficient polarization independent vertical resonant couplers for active-passive monolithic integration," Optics Express, vol. 16, no. 12, pp. 8350-8358, 2008.

[51] V. M. N. Passaro and F. De Leonardis, "Design of polarisation insensitive vertical couplers in silicon-oninsulator waveguides with small cross section," in Proceedings of the 4th IEEE/LEOS Workshop on Fibres and Optical Passive Components (WFOPC '05), vol. 2005, pp. 187-191, Palermo, Italy, June 2005.

[52] C. W. Lee and M. K. Chin, "Design of vertical polarizationmode splitter with mode-size transformer," IEEE Photonics Technology Letters, vol. 16, no. 9, pp. 2069-2071, 2004.

[53] M. K. Chin, C. W. Lee, and J. Shen, "Polarization-independent vertical coupler for photonics integration," Optics Express, vol. 12, no. 1, pp. 117-123, 2004. 
[54] C. W. Lee, M. K. Chin, M. K. Iyer, and A. Popov, "Asymmetric waveguides vertical couplers for polarization-independent coupling and polarization-mode splitting," Journal of Lightwave Technology, vol. 23, no. 4, pp. 1818-1827, 2005.

[55] C. J. Brooks, A. P. Knights, and P. E. Jessop, "Verticallyintegrated multimode interferometer coupler for 3D photonic circuits in SOI," Optics Express, vol. 19, pp. 2916-2921, 2011.

[56] “BeamProp," RSoft, Inc., Version 5.0, 2001.

[57] B. Broberg and S. Lindgren, "Refractive index of In1$x G a x A s y P 1-y$ layers and InP in the transparent wavelength region," Journal of Applied Physics, vol. 55, no. 9, pp. 33763381, 1984.

[58] C.-W. Lee, S.-Y. Lee, and M.-K. Chin, "Demonstration of a single-mesa vertical coupler and its integration with a multimode interferometer," Semiconductor Science and Technology, vol. 26, no. 7, Article ID 075008, 2011.

[59] C. W. Lee, D. Nie, T. Mei, and M. K. Chin, "Study and optimization of room temperature inductively coupled plasma etching of InP using $\mathrm{Cl}_{2} / \mathrm{CH}_{4} / \mathrm{H}_{2}$ and $\mathrm{CH}_{4} / \mathrm{H}_{2}$," Journal of Crystal Growth, vol. 288, no. 1, pp. 213-216, 2006.

[60] T. Feuchter and C. Thirstrup, "High precision planar waveguide propagation loss measurement technique using a Fabry-Perot cavity," IEEE Photonics Technology Letters, vol. 6, no. 10, pp. 1244-1246, 1994.

[61] L. B. Soldano and E. C. M. Pennings, "Optical multi-mode interference devices based on self-imaging: principles and applications," Journal of Lightwave Technology, vol. 13, no. 4, pp. 615-627, 1995. 

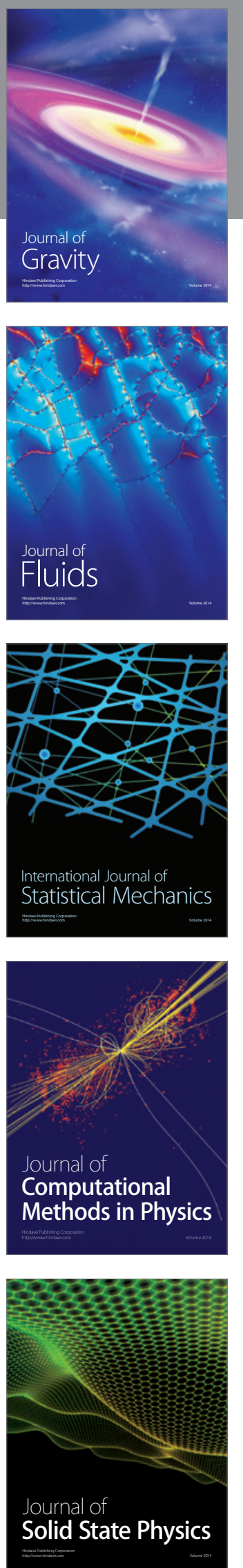

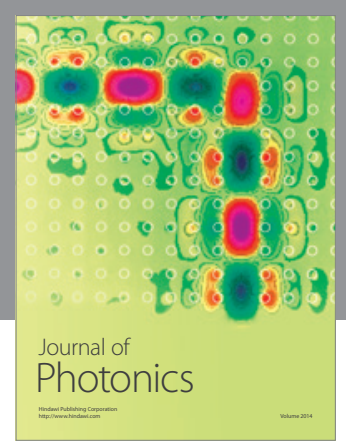

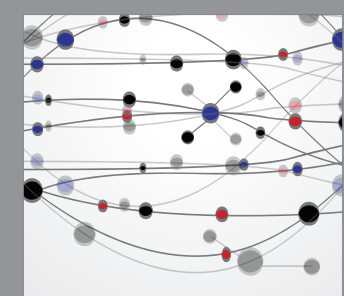

The Scientific World Journal
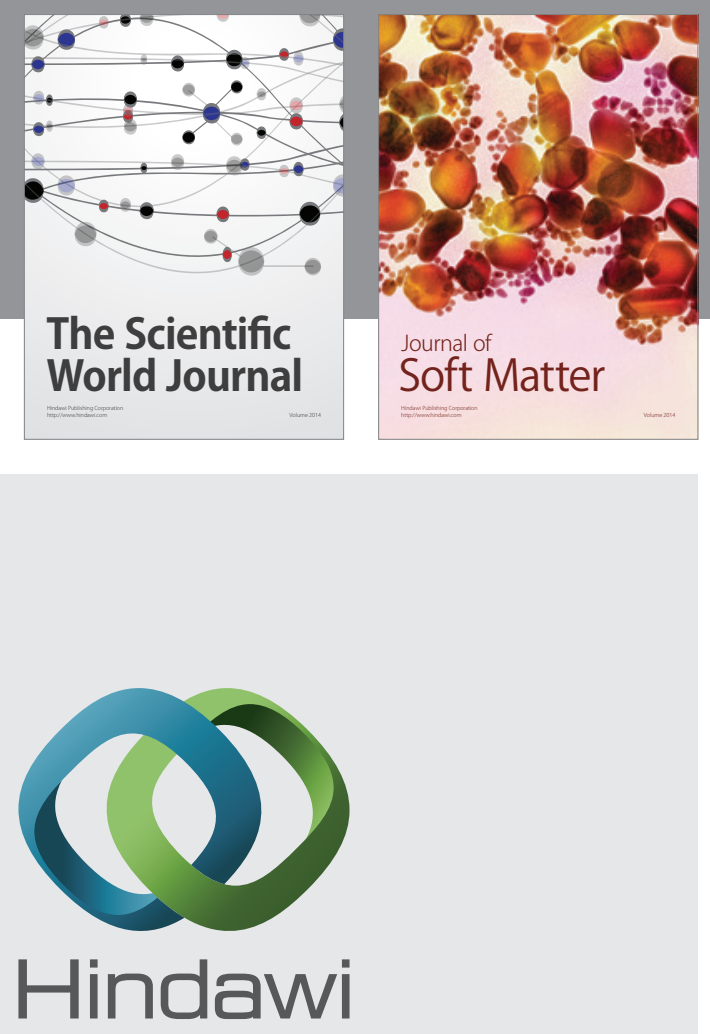

Submit your manuscripts at

http://www.hindawi.com
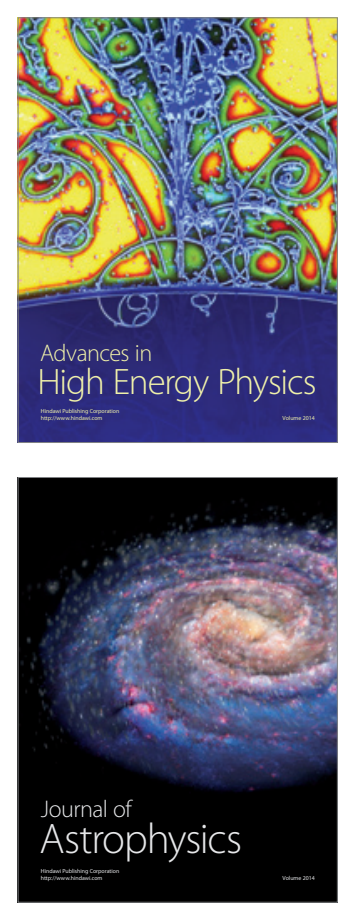
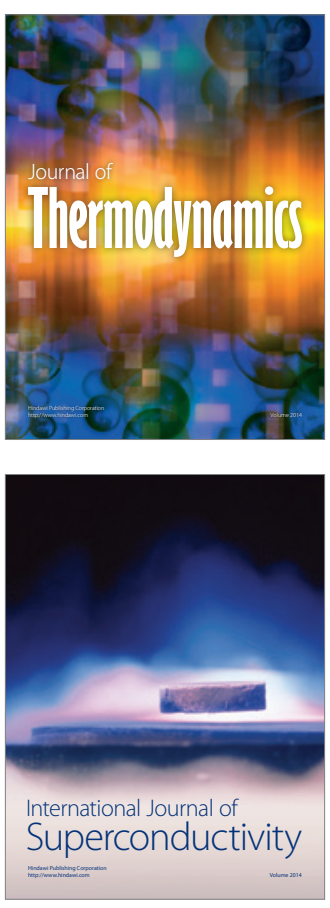
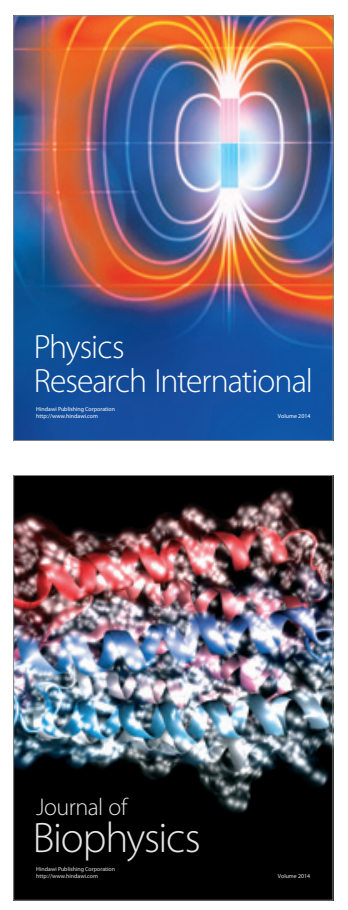
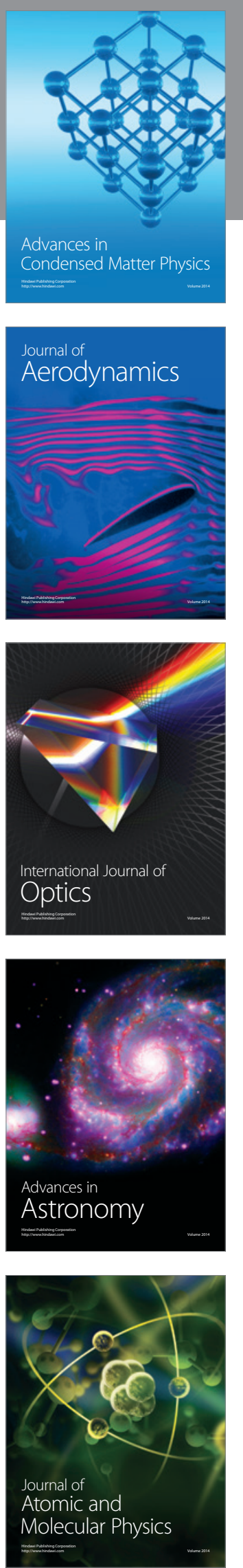